\title{
Upper Bounds on Scattering Processes and Metamaterial-Inspired Structures that Reach Them
}

\author{
Iñigo Liberal, Member, IEEE, Iñigo Ederra, Ramón Gonzalo, Member, IEEE, and \\ Richard W. Ziolkowski, Fellow, IEEE
}

\begin{abstract}
The physical limitations on time-harmonic scattering processes are investigated on the basis of the optical theorem. Previously derived least upper bounds on the total scattering and absorption cross-sections are obtained from it in a straightforward manner. In addition, it reveals a practical upper bound for the bistatic cross-section when evaluated in any direction. It is proved further that the maximum upper bound of the bistatic cross-section occurs in the forward scattering direction and that the corresponding upper bound for the backscattering direction is four times smaller than this maximum value. Metamaterialinspired electrically small antennas and scattering particles that approach these upper bounds are demonstrated. These examples numerically validate the derived upper bounds, as well as illustrate the important physical principles underlying them.
\end{abstract}

Index Terms-Electromagnetic scattering, physical bounds, antenna theory, electrically small antennas.

\section{INTRODUCTION}

Scattering or re-radiation processes in receiving antennas are of paramount importance from both fundamental and technological points of view. The significance of their properties and their consequent impact on applications was already recognized in the seminal book [1], and the related classic papers on minimum scattering antennas [2], [3]. For instance, these works established the fact that antennas with the same gain and radiation pattern can have significantly different scattering properties (see, e.g., [1] p. 317).

Taking advantage of the available degrees of freedom to either minimize or maximize the scattering by a receiving antenna is equally relevant. Minimizing the scattering, for example, impacts the reduction of the overall radar cross section [4], the mitigation of the blockage between neighboring antennas [5], [6], and/or the avoidance of efficiency penalties in near-field wireless power transfer [7]. On the other hand, maximizing the scattering is of interest for radar-based monitoring and/or imaging systems, passive RFID tags [8] and architectural and health monitoring [9].

Receiving antennas are not physically different from any other scatterer. In particular, while there is a certain degree of

I. Liberal, I. Ederra and R. Gonzalo are with the Electrical and Electronic Engineering Department, Universidad Pública de Navarra, Pamplona, Spain, e-mail: inigo.liberal@unavarra.es

R. W. Ziolkowski is with the Department of Electrical and Computer Engineering, University of Arizona, Tucson, AZ, 85721 USA, email:ziolkowski@ece.arizona.edu

This work was supported in part by the Spanish Ministry of Science and Innovation, Dirección General de Investigación y Gestión del Plan Nacional de I+D+I, Subdirección General de Proyectos de Investigación, Project Nos. CSD2008-00066 and TEC2013-47753-C3-1-R, and by NSF contract number ECCS-1126572.

Manuscript received May. XX, 2014; revised ... freedom in tailoring the scattering by a receiving antenna, there are also fundamental limitations. For instance, as emphasized by the optical theorem [10], [11], energy conservation imposes the fact that absorption processes in far-field interactions inevitably must be accompanied by scattering. This intrinsic property limits the effective area of an antenna as a function of its visibility. ${ }^{1}$ Moreover, even in the ideal limit of no material dissipation, scattering processes encompass the scattering resistance (see, e.g., [12]), which damps the excitation of the currents in the scatterer and, hence, ultimately limits the scattered power [12], [13].

Motivated by these physical constraints, upper bounds on the powers extracted, scattered and absorbed from timeharmonic fields by scatterers/receiving antennas have been derived based on multipolar [7], [14], algebraic [13] and circuit model [12] approaches. Yet another methodology is introduced in this work to investigate the physical limitations on the scattering of time-harmonic fields. Specifically, it will be demonstrated that all previous bounds can be derived directly from the optical theorem (see, e.g., [10], [11]) in a strikingly simple manner. This optical theorem-based approach also enables the derivation of novel upper bounds on the bistatic cross-section, i.e., the amount of power that can be re-radiated in a specific direction. Note, however, that the derivation will be restricted to obstacles illuminated by propagating planewaves.

These bounds are particularly relevant from a practical standpoint since, despite the fundamental interest in the absorbed and scattered powers, the bistatic cross-section is the quantity of interest for most engineering systems. Note that while the extracted power can be indirectly measured through the forward scattering [15], discriminating between the absorption and scattering contributions requires the recording of the scattered fields in all directions, which is seldom, if ever, truly feasible. In contrast, most sensing and imaging systems illuminate their samples along a given direction, $\widehat{\mathbf{k}}_{i}$, and then measure the scattered field along a single direction, $\widehat{\mathbf{r}}$. A natural choice is to measure the backscattering or reflection from the sample, i.e., setting $\widehat{\mathbf{r}}=-\widehat{\mathbf{k}}_{i}$. Thus, the monitoring device is included with the source in a single transmit-receive antenna system.

A deeper understanding of the physical limitations on scattering is a necessary initial step towards the design of more efficient receiving antennas that either maximize or

\footnotetext{
${ }^{1}$ As noted in [7], this limitation can be circumvented in near-field interactions, where the receiving antenna can modify the power supplied by the sources.
} 


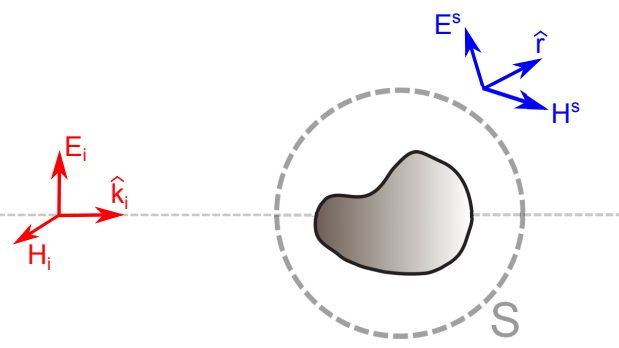

Fig. 1. Geometry of the scattering problem: finite-size object enclosed within the surface $A$, illuminated by a plane-wave propagating along the $\widehat{\mathbf{k}}_{i}$ direction.

minimize their scattering processes. In particular, it will be shown that forward-scattering receiving antennas are optimal for maximizing the scattered and absorbed powers, as well as for the bistatic cross-section. Several electrically small, forward-scattering receiving antennas and scattering particles that are based on metamaterial-inspired design concepts [16] are introduced to illustrate and to validate numerically these outcomes. Moreover, the designs selected for this discussion actually approach the upper bounds and thus reveal several of the basic principles needed to optimize general designs.

\section{Geometry And Definitions}

As a generic far-field scattering problem, consider a scatterer illuminated by a time-harmonic plane-wave that is linearly polarized along the $\widehat{\mathbf{e}}_{i}$ direction and propagating along the orthogonal $\widehat{\mathbf{k}}_{i}$ direction (c.f., Fig. 1). With the $e^{j \omega t}$ time convention, the incident electric field can be written as follows

$$
\mathbf{E}^{i}(\mathbf{r})=\widehat{\mathbf{e}}_{i} E_{0} e^{-j k_{0} \widehat{\mathbf{k}}_{i} \cdot \mathbf{r}}
$$

where $k_{0}$ is the wave number in the homogeneous region outside the scatterer. The total electric field is given by the superposition of this incident field and the scattered field, i.e., the field re-radiated by the scatterer, as: $\mathbf{E}^{t}=\mathbf{E}^{s}+\mathbf{E}^{i}$. In general, the scattered field can be of any form; it is a complex function of the properties of the scatterer. However, for finitesize objects, the scattered electric field in the far-zone reduces to a spherical wave of the form:

$$
\lim _{r \rightarrow \infty} \mathbf{E}^{s}(\mathbf{r})=E_{0} \frac{e^{-j k_{0} r}}{k_{0} r} \mathbf{F}(\widehat{\mathbf{r}})
$$

where $\mathbf{F}(\widehat{\mathbf{r}})$ is the dimensionless (normalized to the incident field magnitude) far-field vector scattering pattern along the direction $\widehat{\mathbf{r}}$. The set of powers scattered, absorbed and extracted by the scatterer, as well as their respective normalized (total) cross-sections are defined as follows (see, e.g., [17])

$$
\begin{array}{rlrl}
P_{\mathrm{scat}} & =\oiint_{A} \mathbf{S}^{s} \cdot \widehat{\mathbf{n}} d A, & \sigma_{\mathrm{scat}} & =\frac{P_{\mathrm{scat}}}{S^{i} \lambda^{2}} \\
P_{\mathrm{abs}}=-\oiint_{A} \mathbf{S}^{t} \cdot \widehat{\mathbf{n}} d A, & \sigma_{\mathrm{abs}}=\frac{P_{\mathrm{abs}}}{S^{i} \lambda^{2}} \\
P_{\text {ext }}=P_{\mathrm{abs}}+P_{\mathrm{scat}}, & \sigma_{\mathrm{ext}}=\frac{P_{\mathrm{ext}}}{S^{i} \lambda^{2}}
\end{array}
$$

with $\widehat{\mathbf{n}}$ being the unit vector normal to the surface $A$ which encloses the scatterer. $\mathbf{S}^{u}=\frac{1}{2} \operatorname{Re}\left\{\mathbf{E}^{u} \times\left(\mathbf{H}^{u}\right)^{*}\right\}$, for $u=$ $i, s, t$, is the Poynting vector field associated with the incident, scattered and total fields, respectively. The incident power density is $S^{i}=\widehat{\mathbf{k}}_{i} \cdot \mathbf{S}^{i}$. For a lossless receiving antenna, all of the absorption is identified with the power collected in its port. Thus, the absorption cross-section and the antenna gain can be related in this idealized case by invoking reciprocity (see, e.g., [18]) as $G=4 \pi \sigma_{\text {abs }}$, where $G$ denotes the realized antenna gain for the polarization $\widehat{\mathbf{e}}_{i}$ and direction $\widehat{\mathbf{k}}_{i}$.

All of the aforementioned figures of merit describe the overall balance of powers involved in the scattering problem. By contrast, the bistatic cross-section: $\sigma_{\mathrm{b}}(\widehat{\mathbf{r}})$, is the usual figure of merit to describe the amount of power scattered along a given direction $\widehat{\mathbf{r}}$. It is defined as follows [17]

$$
\sigma_{\mathrm{b}}(\widehat{\mathbf{r}})=\sigma_{\text {scat }} D_{\text {scat }}(\widehat{\mathbf{r}})
$$

where $D_{\text {scat }}(\widehat{\mathbf{r}})$ is the scattering directivity along the direction $\widehat{\mathbf{r}}$ and is expressed as:

$$
D_{\text {scat }}(\widehat{\mathbf{r}})=\lim _{r \rightarrow \infty} 4 \pi r^{2} \frac{\widehat{\mathbf{r}} \cdot \mathbf{S}^{s}(\widehat{\mathbf{r}})}{P_{\text {scat }}}=4 \pi \frac{|\mathbf{F}(\widehat{\mathbf{r}})|^{2}}{\iint|\mathbf{F}(\widehat{\mathbf{r}})|^{2} d \Omega}
$$

\section{OpticAl TheOREM AND Associated PhysiCAL BOUNDS}

The optical theorem is a classical result that relates the extinction cross-section to the imaginary part of the forwardscattering amplitude that is co-polarized with the incident field [10]

$$
\sigma_{\text {ext }}=\sigma_{\text {abs }}+\sigma_{\text {scat }}=\frac{1}{\pi} \operatorname{Im}\left\{\widehat{\mathbf{e}}_{i} \cdot \mathbf{F}\left(\widehat{\mathbf{k}}_{i}\right)\right\}
$$

It arises as a direct consequence of energy conservation, i.e., the power extracted by a scatterer from the incident field must be removed by means of destructive interference. This extraction, in turn, requires a certain amount of the incident field to be scattered into its direction of propagation. Thus, the imposition a certain scattered field enforces a correlation between the absorption and scattering processes, whose powers combine to yield the extracted power.

As demonstrated in [11], the following inequality can be derived by simply noting that the imaginary part of the right hand side of (8) is less than or equal to its absolute value:

$$
\sigma_{\text {ext }}=\sigma_{\text {abs }}+\sigma_{\text {scat }} \leq \sqrt{\frac{1}{\pi} D_{\text {scat }}\left(\widehat{\mathbf{k}}_{i}\right) \sigma_{\text {scat }}}
$$

This inequality was employed extensively in [11] to investigate the absorption efficiency of a receiving antenna, usually defined as $\eta_{\text {abs }}=\sigma_{\text {abs }} /\left(\sigma_{\text {abs }}+\sigma_{\text {scat }}\right)$, and the limitations on the gain of a receiving antenna as a function of this absorption efficiency. We have found that by manipulating Eq. (22) in [11], the limitation on the receiving antenna gain can be written in an elucidating manner as a function of its absorption efficiency $\eta_{\text {abs }}$ :

$$
G \leq D_{\text {scat }}\left(\widehat{\mathbf{k}}_{i}\right) 4 \eta_{\text {abs }}\left(1-\eta_{\text {abs }}\right)
$$

Eq. (10) clearly illustrates how the antenna gain of a minimum scattering (cloaked) sensor must be ultimately reduced when its scattering is minimized. It also emphasizes how the receiving antenna's ability to channel its scattering into the forward 
direction, i.e., its forward directivity: $D_{\text {scat }}\left(\widehat{\mathbf{k}}_{i}\right)$, increases the range of its achievable gain.

We also can demonstrate that the inequality (9) derived in [11] can be manipulated to reproduce the upper bounds on the scattered and absorbed powers presented in [7], [12]-[14] in a simple manner for the restricted cases under consideration here, i.e., when the incident electromagnetic field is a propagating plane wave. It also enables the derivation of novel bounds on the bistatic cross-section for individual observation angles.

In particular, it directly follows from Eq. (9) that $\sigma_{\text {scat }}$ is maximized when the absorption cross-section is zero $\left(\sigma_{\mathrm{abs}}=\right.$ 0 ). Physically, this conclusion is consistent with the fact that dissipation losses would only damp the excitation of currents in the antenna. Thus, an upper bound on the scattering crosssection can be derived simply by taking the lossless case $\left(\sigma_{\mathrm{abs}}=0\right)$ in (9) and reorganizing the terms to obtain:

$$
\sigma_{\text {scat }} \leq \frac{1}{\pi} D_{\text {scat }}\left(\widehat{\mathbf{k}}_{i}\right)
$$

In order to maximize the absorption cross-section (absorbed power), it is sufficient to move $\sigma_{\text {scat }}$ to the right hand side of (9), take derivatives with respect to $\sigma_{\text {scat }}$, and set the result to zero. In this manner, it follows that $d \sigma_{\text {abs }} / d \sigma_{\text {scat }}=0$ is achieved when $\sigma_{\text {scat }}=D_{\text {scat }}\left(\widehat{\mathbf{k}}_{i}\right) /(4 \pi)$. Alternatively, the upper bound of $\sigma_{\text {abs }}$ can be obtained by introducing the inequality of geometric means: $\sigma_{\text {abs }}+\sigma_{\text {scat }} \geq 2 \sqrt{\sigma_{\text {abs }} \sigma_{\text {scat }}}$, into (9) and then cancelling out the $\sigma_{\text {scat }}$ terms. In either case, it is found that the absorption cross section is upper bounded by

$$
\sigma_{\text {abs }} \leq \frac{1}{4 \pi} D_{\text {scat }}\left(\widehat{\mathbf{k}}_{i}\right)
$$

It is worth noting that the absorption and scattering crosssections are equal, $\sigma_{\text {abs }}=\sigma_{\text {scat }}$, when the absorption crosssection is maximized, i.e., when $\sigma_{\mathrm{abs}}=D_{\text {scat }}\left(\widehat{\mathbf{k}}_{i}\right) /(4 \pi)$. This condition can be intuitively understood as a conjugate matching condition when the scattering problem is represented by equivalent circuit models [12], [13], [18].

Equivalently to Eq. (12), it can be stated that the maximal gain of a lossless (except into the port) receiving antenna is smaller than or equal to the forward-scattering directivity, i.e.,

$$
G_{\text {lossless }} \leq D_{\text {scat }}\left(\widehat{\mathbf{k}}_{i}\right)
$$

Note that this statement is conceptually different from the well-known fact that the gain of an antenna is always smaller than or equal to its radiation directivity, i.e., since by definition, $G=$ efficiency $\times \mathrm{D}_{\mathrm{rad}}$, one has $G \leq D_{\mathrm{rad}}$. To understand this fact, it is important to distinguish between the radiation, $D_{\text {rad }}$, and scattering, $D_{\text {scat }}$, directivities. The former describes the directivity associated with the fields generated when an antenna is fed at its ports, i.e., its radiation mode [18]. The latter is the directivity associated with the fields re-radiated (scattered) by the antenna in its receiving mode, e.g., when the antenna is illuminated by an external plane-wave [11]. Note that the current distributions excited in the radiation and receiving modes are in general different [19], which in turn leads to the different radiation and scattering directivities. In a similar manner, if the antenna is composed of reciprocal materials, it can also be stated that the gain in the receiving mode is upper bounded by the directivity in the radiation mode. In direct contrast, Eq. (13) upper bounds the gain in the receiving mode by the scattering directivity in the forward direction, i.e., $G \leq D_{\text {scat }}\left(\widehat{\mathbf{k}}_{i}\right)$.

The upper-bounds (11)-(12) emphasize how both absorption and scattering are limited by the ability of the obstacle to concentrate the scattered (re-radiated) field along the direction of propagation of the incident field. Theoretically, the directivity in the forward direction, $D_{\text {scat }}\left(\widehat{\mathbf{k}}_{i}\right)$, is unbounded even for a finite-size scatterer. The same is true for the absorption and scattering cross-sections [20]. However, these superdirective behaviors come at the cost of increasingly narrower bandwidths and significant sensitivity to fabrication tolerances. Note that the bounds (11)-(12) have been established for a purely time-harmonic (single frequency) incident field. When integrated over all frequencies, the resulting total absorption and scattering cross-sections must be consistent with their bounds as derived in [21]-[26].

Alternatively, one might wonder how one could attain a particular scattering cross-section behavior from one or more canonical responses of the scatterer. For example, when considering the response of a scatterer to the excitation of one or more spherical harmonics, it has been shown that each electric or magnetic multipole of order $n$ increases the maximum directivity by a factor $n+\frac{1}{2}$ [27], [28]. Equivalently, it can be stated that if one can excite electric and magnetic multipoles within a scatterer up to a maximum order $N$, its response features a maximal directivity: $D_{\text {scat }}^{\max }\left(\widehat{\mathbf{k}}_{i}\right)=N^{2}+2 N$. It is also worth noting that substituting this maximal directivity into (11)-(12), one finds that these inequalities are equivalent to the upper bounds on the extracted and scattered powers introduced in [7], [12]-[14]. Thus, the upper bounds presented in those articles can be independently derived from the inequality (9) presented in [11], which is a direct consequence of the optical theorem, in a strikingly simple manner. However, to re-emphasize, this simpler derivation is restricted to incident fields consisting of propagating plane-waves. Despite this limitation, the present demonstration does not assume an invertible polarizability matrix. Therefore, it holds for the singular cases of scattering particles, i.e., for a particle having a non-invertible polarizability matrix. These singular cases were excluded from the algebraic demonstration presented for the extracted and scattered powers in [13].

Once the limits on the integrated scattering responses, i.e., those which were obtained by integrating over all $\widehat{\mathbf{r}}$ directions, have been analyzed, one might wonder how large the scattering into a specific direction can be. At first sight, one might conclude that in order to maximize the scattering along a given direction $\widehat{\mathbf{r}}$, the scattering pattern should be as directive as possible for that direction. However, by introducing (11) into (6), the following upper bound on the bistatic cross-section can be derived

$$
\sigma_{\mathrm{b}}(\widehat{\mathbf{r}}) \leq \frac{1}{\pi} D_{\text {scat }}(\widehat{\mathbf{r}}) D_{\text {scat }}\left(\widehat{\mathbf{k}}_{i}\right)
$$

Eq. (14) illustrates that in order to maximize the bistatic cross-section, it is necessary instead to design a scatterer 
with two directive beams: one pointing towards the desired direction, $\widehat{\mathbf{r}}$, and another one pointing along the direction of propagation of the incident field, $\widehat{\mathbf{k}}_{i}$. This constraint imposes severe limitations on the achievable bistatic cross-sections. This is particularly true for electrically small particles, for which obtaining two simultaneous directive beams is a very challenging task. Bearing in mind this limitation, the normalized bistatic cross-section is maximized when $\widehat{\mathbf{r}}=\widehat{\mathbf{k}}_{i}$, i.e., when the direction of incidence and the measurement direction are equal. In such a case, the upper bound of the bistatic crosssection is found by introducing the maximum directivity into (14), which leads to the upper bound:

$$
\sigma_{\mathrm{b}}\left(\widehat{\mathbf{k}}_{i}\right) \leq \frac{1}{\pi} D_{\text {scat }}^{2}\left(\widehat{\mathbf{k}}_{i}\right) \leq \frac{1}{\pi}\left(N^{2}+2 N\right)^{2}
$$

The upper bound in any other direction will be smaller. For example, by using Eq. (14) and a multipolar analysis of the forward-backward directivity product, the following upper bound for the backscattering cross-section can be derived

$$
\sigma_{\mathrm{b}}\left(-\widehat{\mathbf{k}}_{i}\right) \leq \frac{1}{4 \pi}\left(N^{2}+2 N\right)^{2}
$$

For the sake of brevity, the details of the mathematical derivation of Eq. (16) have been included as supplementary material. The derivation is even more complicated for an arbitrary direction and any given number of multipoles.

It is evident from Eqs. (15) and (16) that the maximal backscattering is four times smaller than the maximal bistatic cross-section, i.e., the forward scattering cross-section. Therefore, it reveals that the price to be paid for using a single transmit-receive, monostatic interrogation system rather than the optimum, forward-scattering bistatic one is minus $6 \mathrm{~dB}$ (factor of 4). In addition, it is demonstrated in the supplementary material (refer to Eqs. (35) and (36) and the associated discussion) that the bound (16) can be reached with either electric or magnetic multipoles only, it demonstrates that, in terms of backscattering, there is no benefit derived from the simultaneous excitation of electric and magnetic dipoles, nor from the coupling between the different multipoles via non-reciprocal and/or magnetoelectric coupling phenomena. The fact that the optimal backscattering configuration can be constructed with only electric multipoles is a positive outcome, particularly at high frequencies, where the magnetic response of matter is inherently weak (see, e.g., [29]).

Eqs. (14)-(16) are the main analytical results of this work. They represent the upper bounds on the achievable scattering towards a specific direction that, to the best knowledge of the authors, are presented here for the first time. Due to their fundamental nature, they have far-reaching implications in spectroscopy, radar, sensing and imaging technologies. Moreover, they clarify the limits on the performance of any system that relies on measuring the scattering of a given object along a specific direction.

\section{Electrically SMALl ForWARD-SCATtERING PARTICLES}

The optical theorem-based bounds provide a deeper insight into the physical mechanisms that limit the absorption and scattering processes. Thus, they are invaluable when one wants to identify strategies for the design of scatterers/receiving antennas that might approach them. Specifically, these upper bounds suggest that a scatterer/receiving antenna that concentrates its scattering into the forward direction is optimal to maximize the received power (12); the visibility, i.e., both the overall scattering cross-section (11) and the scattering crosssection in a specific direction (14); and the performance as a cloaked sensor (10). Under this perspective, we discuss the design and performance characteristics of electrically small forward-scattering particles, antennas, and/or sensors based on metamaterial-inspired structures. The design of scatterers that maximize the backscattering, as suggested by Eq. (16), is left for future efforts.

We emphasize that the response of an electrically small particle can be approximated by the combination of its electric and magnetic dipole moments. In such a case, the maximum directivity is achieved by the balanced combination of perpendicular electric and magnetic dipole moments [30], which is usually termed as a Huygens source. Therefore, the Huygens source directivity in the forward direction $D_{\text {scat }}\left(\widehat{\mathbf{k}}_{i}\right)=3$ can be introduced in the bounds (10)-(14) to establish a reference/baseline case for electrically small devices. We will denote it here as an ideal Huygens source particle/antenna.

In theory, an unlimited superdirective response can be obtained by exciting higher order modes (HOMs) [31], [32]. However, practical implementations of HOM approaches are hindered by increasingly narrower bandwidths; the fact that they are ill-posed solutions and, hence, are extremely sensitive to fabrication tolerances; and the dramatic damping that occurs when realistic losses are included [18], [33], [34]. Therefore, we will adhere here to the strategy of enhancing the directivity to reach the upper bounds by achieving a balanced combination of perpendicular electric and magnetic dipole moments, i.e., emulating a Huygens source.

The simplest implementation of a Huygens source scattering particle could consist of an electrically small body with large, low-loss and equal permittivity and permeability [35]. However, to the best of the authors knowledge, materials fulfilling these three requirements are yet unknown. At optical frequencies, Huygens source nanoparticle lasers have been designed by using a combination of plasmonic and semiconductor layers [36]. At microwave frequencies, successful implementations of Huygens source antennas have been carried out based on metamaterial-inspired concepts [16], [37] and chiral particles [38], [39]. Here we will examine the scattering properties of Huygens source antenna designs whose radiation properties were investigated in [16], [37], as well as further evolutions of those metamaterial-inspired scatterers, to illustrate how closely they can approach the derived upper bounds.

\section{A. Coin Scattering Particle}

We begin with a particle that maximizes the scattering in the forward direction. Following the philosophy of combining perpendicular electric and magnetic dipoles, we propose the use of a coin-like particle as schematically depicted in Fig. 2. This coin particle consists of a cylindrical dielectric substrate 


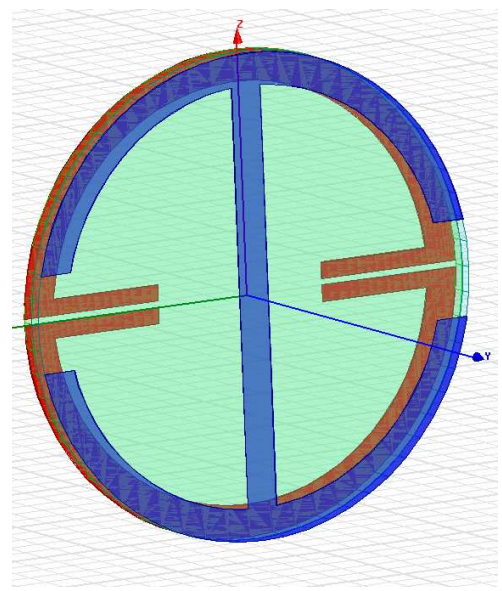

(a)

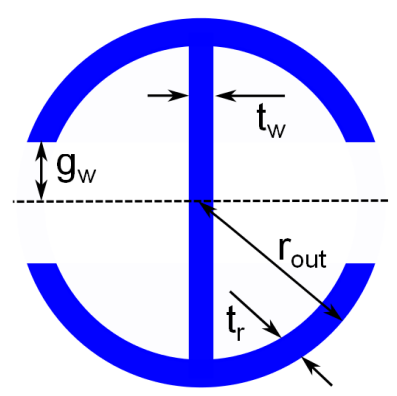

(c)

$$
\begin{gathered}
r_{\mathrm{out}}=15 \mathrm{~mm} \\
t_{\mathrm{w}}=t_{\mathrm{r}}=2 \mathrm{~mm} \\
g_{\mathrm{w}}=3 \mathrm{~mm} \\
t_{\mathrm{s}}=5.15 \mathrm{~mm} \\
t_{\mathrm{g}}=1 \mathrm{~mm} \\
g_{\mathrm{s}}=0.4 \mathrm{~mm}
\end{gathered}
$$

(b)

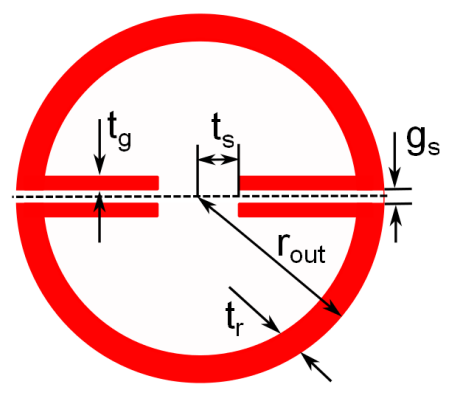

(d)
Fig. 2. Coin particle geometry: (a) HFSS model, (b) dimensions, and sketches of its (c) electric and (d) magnetic resonators.

having electric and magnetic dipole resonators imprinted on each of its sides. The substrate consists of two layers of $0.787 \mathrm{~mm}$ thick Rogers Duroid RO5880 with both resonators being constructed with copper traces having $0.07 \mathrm{~mm}$ thickness. The magnetic resonator is a pair of capacitively loaded loops, whereas the electric resonator is a top-hat loaded dipole. Despite the fact that both resonators are intrinsically coupled, the frequency position of their resonances can almost be tuned independently by modifying their respective loads, i.e., the widths and lengths of the traces and gaps of both elements, which enables a tailored superposition of the electric and magnetic dipole resonances in a straightforward manner. While this optimization process is not reported here for the sake of brevity, the resulting geometry is detailed in Fig. 2. Note that the design features an external radius of $r_{\text {out }}=15 \mathrm{~mm}$ $\left(k_{0} r_{\text {out }}=0.47\right.$ at $\left.1.5 \mathrm{GHz}\right)$. Consequently, it can be regarded fairly as an electrically small resonator.

Fig. 3 presents the HFSS-simulated values of the coin particle normalized bistatic cross-section in the forward direction, $\sigma_{b}\left(\widehat{\mathbf{k}}_{i}\right)$, as a function of frequency, when the particle is illuminated with a $\widehat{\mathbf{z}}$-polarized plane-wave propagating along the $\widehat{\mathbf{x}}$ direction. In order to establish a reference framework, the figure also includes the performance of the same particle implemented with lossless materials (i.e., replacing the copper strips with a PEC material and removing the losses in the dielectric substrate); the upper bound (15); and the hypothetical performance of an ideal Huygens particle.

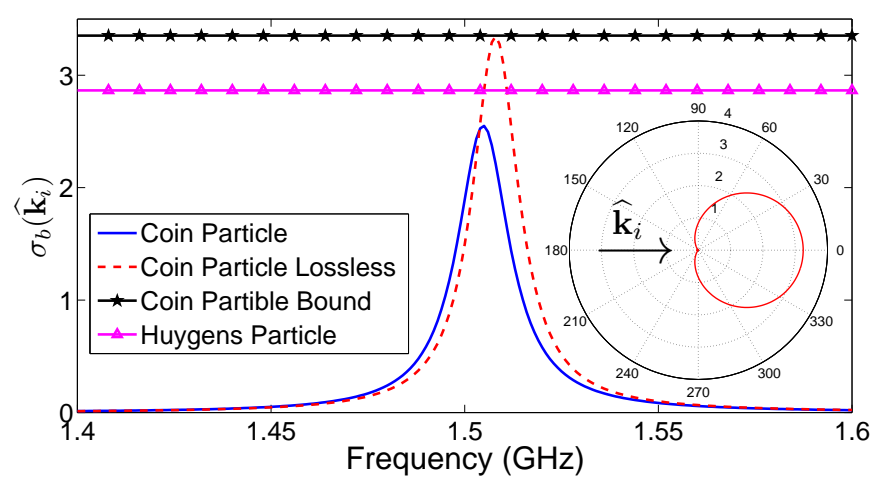

Fig. 3. Bistatic cross-section in the forward direction, $\sigma_{b}\left(\widehat{\mathbf{k}}_{i}\right)$, for the coin particle, its lossless implementation, the upper bound (15), and an ideal Huygens particle. Inset: Coin particle's scattering directivity pattern in the $\mathrm{H}$-plane at $1.505 \mathrm{GHz}$.

It can be concluded from Fig. 3 that the $\sigma_{b}\left(\widehat{\mathbf{k}}_{i}\right)$ spectra of both the coin particle and its lossless counterpart are each characterized by a single resonant peak which occur, respectively, at $1.505 \mathrm{GHz}$ and $1.508 \mathrm{GHz}$. The existence of a single resonant peak suggests that both the electric and magnetic resonances are overlapping, and, indeed, this can be ratified by inspecting the inset of Fig. 3, which depicts the scattering directivity pattern of the coin particle in the $\mathrm{H}$ plane at its resonance frequency, $1.505 \mathrm{GHz}$. It is apparent that the coin particle features a Huygens source-like pattern that concentrates the scattered field into the forward direction. The front-to-back ratio (FTBR) is larger than $100(20 \mathrm{~dB})$, and the maximal directivity $D_{\text {scat, } \max }\left(\widehat{\mathbf{k}}_{i}\right)=3.245$ is actually slightly larger that 3.0, the ideal Huygens particle value. This outcome is due to the fact that the effective lengths of the electric and magnetic dipole resonators are somewhat larger than those of the infinitesimal electric and magnetic dipoles constituting the ideal Huygens source. Consequently, the maximal bistatic cross-section according to the bound (15) is $\sigma_{b}\left(\widehat{\mathbf{k}}_{i}\right) \leq(3.245)^{2} / \pi \simeq 3.352$. Note that this value is very closely approached by the peak of the lossless implementation: $\sigma_{b}\left(\widehat{\mathbf{k}}_{i}\right)=3.347$. In fact, the forward bistatic cross-section of the ideal lossless coin particle is even slightly larger than that of an ideal Huygens source particle, i.e, $\sigma_{b}\left(\widehat{\mathbf{k}}_{i}\right)=3^{2} / \pi \simeq 2.865$

These simulation results suggest that the ideal coin particle is an optimal strategy for maximizing the bistatic cross-section, and that it also serves as a good test of the validity of the upper bound (15). Notice, however, that the more realistic implementation of the coin particle with copper strips and commercially available substrates inevitably lies below this theoretical value. Specifically, the damping introduced by the dissipation losses reduces the peak value of the bistatic cross-section to $76 \%$ of the upper bound. While it can be considered a remarkable performance for such an electrically small resonator, this result illustrates the difficulties involved in the design of efficient electrically small radiators. Thus, while we have demonstrated that the top theoretical performance in terms of the bistatic cross-section can be obtained by means of 
relatively simple resonators, these results also underscore the fact that there are current practical challenges associated with finding robust materials and techniques that enable reducing the device size while minimizing the damping induced by dissipation losses. It is is also important to emphasize that any implementation relying on the excitation of HOMs would be even more dramatically penalized by the presence of losses. Consequently, these results further suggest that electrically small designs based only on dipolar responses are the most reliable strategy to reach the upper bounds from a practical standpoint.

\section{B. Coin Receiving Antenna: Single Port}

Because of its copper and dielectric losses, the coin particle has a non-negligible absorption cross-section. As is commonly done in the analysis of resonant scattering particles [12], one could tune the induced currents in the coin particle by modifying its configuration and composition, notably its loss tangent, to achieve maximal absorption. In fact, since the losses are more or less uniformly distributed within the structure when it is electrically small, the current distribution is quite insensitive to changes in the conductivity. This property enables the adjustment of the absorption while keeping the scattering pattern, which is controlled by the current distribution, intact. On the other hand, the design of a receiving antenna to maximize its response is significantly more complex. Receiving antennas do discriminate between received and parasitically dissipated powers. Consequently, the goal is to maximize the power absorbed within a specific area (the load), while minimizing the absorption in the rest of the device.

As an example of a proper receiving antenna, consider the design schematically depicted in Fig. 4(a). It is the receiving counterpart of the electrically small Huygens source introduced in [37]. The design consists of the coin particle with a dipole antenna printed in the middle of the two substrate layers. This internal dipole antenna is near-field coupled to both resonators. It converts a portion of the incident wave captured by the resonators into voltages and currents along its coplanar stripline output transmission line, which is terminated with a lumped port having a $50 \Omega$ intrinsic impedance. The details of the geometry of the internal dipole are illustrated in Fig. 4(b). The substrate, as well as the electric and magnetic resonators, are the same as those in Fig. 2, except that now the length of the loop capacitors is characterized by $t_{\mathrm{s}}=5.7 \mathrm{~mm}$.

The HFSS-simulated absorption and scattering properties of the coin receiving antenna are reported in Fig. 5. As shown in Fig. 5(a), the scattering directivity pattern at its resonant frequency, $1.473 \mathrm{GHz}$, is again the familiar Huygens source cardioid pattern. By design, it is a balanced combination of the electric and magnetic dipole resonators and, hence, concentrates the scattering into the forward direction. It has a FTBR that is larger than $100(20 \mathrm{~dB})$ and a maximal directivity, $D_{\text {scat }}=3.211$. Again, this maximum value is slightly larger than that which can be obtained with the ideal Huygens antenna constructed with infinitesimal dipoles. Fig. 5(b) presents the gain - absorption efficiency pairs $\left(G, \eta_{\text {abs }}\right)$ for

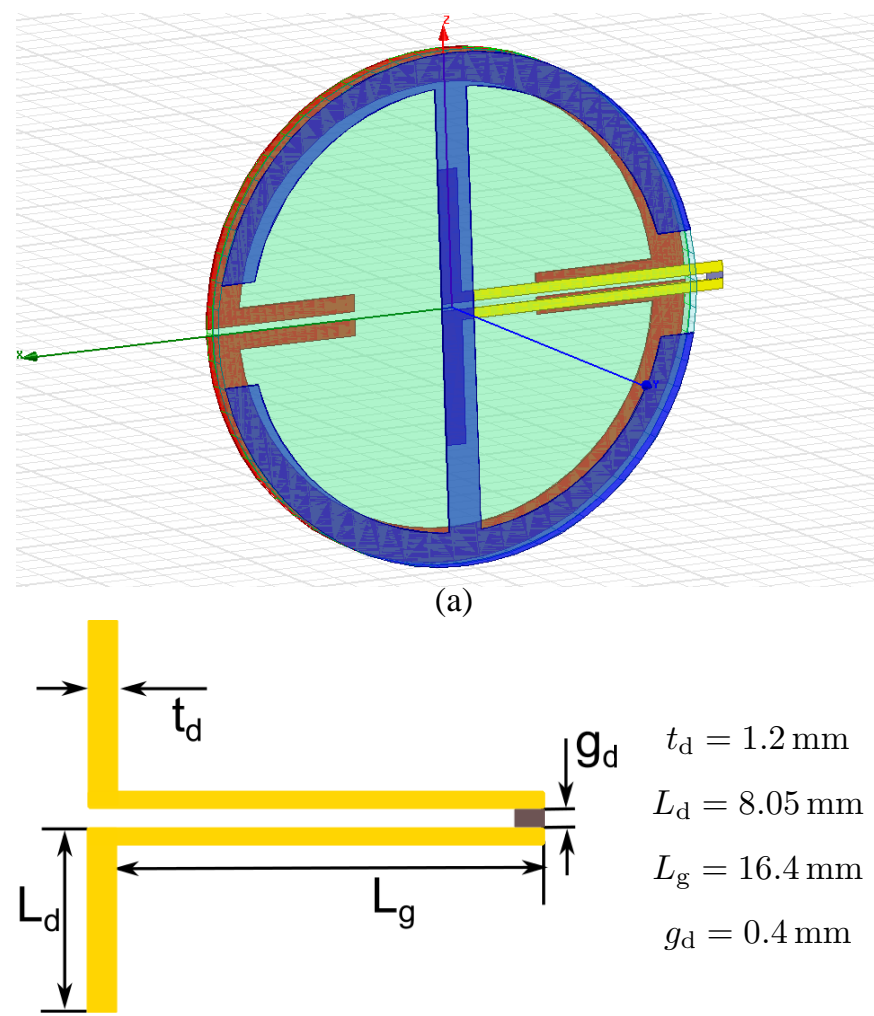

(b)

Fig. 4. HFSS model of the coin antenna. (a) 3D view; (b) Details of the internal dipole antenna geometry.

excitation frequencies ranging from $1.46 \mathrm{GHz}$ to $1.49 \mathrm{GHz}$. For comparison purposes, the figure also includes the lossless implementation of the coin antenna, the upper bound (10), and the performance of an ideal Huygens particle. It is apparent that the lossless implementation of the coin antenna approaches the upper bound (10) very closely (see the inset of Fig. 5(b) for a zoom in of the main plot). This means the coin antenna maximizes the absorbed power without producing any more scattering than would be allowed by the optical theorem. Moreover, it also suggests that the optimal performance of a cloaked sensor can be approached with these metamaterialinspired antenna designs. In this sense, the coin antenna introduced in this study can be considered as an optimal minimum scattering sensor. Consequently, it could be employed, for example, as a minimally disruptive probe. Moreover, since it features a backscattering null, it would help to minimize the interactions between it, as a probe, and any sample.

Note that the performance of a realistic implementation of the coin antenna is degraded away from the upper bound by the presence of unwanted absorption in both the copper and dielectric pieces. Specifically, its peak antenna gain is degraded from an ideal value of 3.207 to 2.709. Again, while a gain of 2.709 is a remarkable value for an electrically small receiving antenna, this result nevertheless emphasizes the great challenges an antenna engineer faces to avoid unwanted absorption when dealing with such an electrically small device. It is also worth noting that the absorption efficiency of the antenna in Fig. 5 is close to 50\%, as it must be for maximal absorption [7], [12]. In fact, the antenna gain, $G=3.207$, 


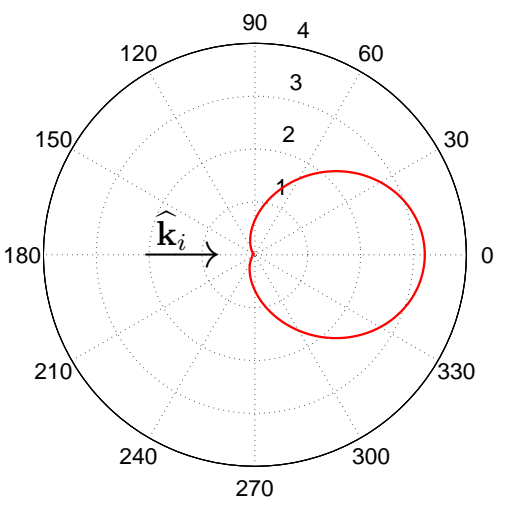

(a)

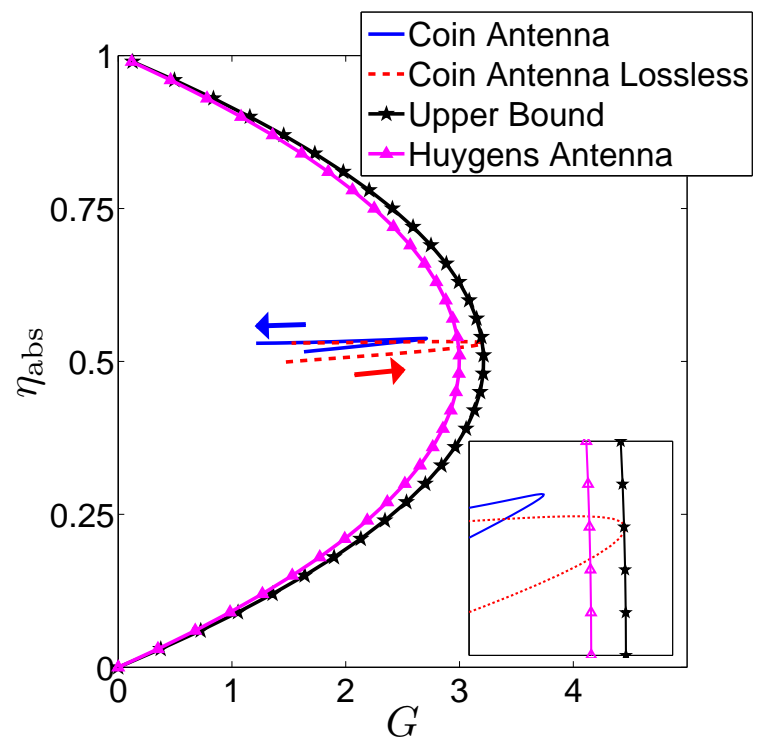

(b)

Fig. 5. HFSS simulated performance of the coin antenna. (a) H-plane scattering directivity pattern at its resonant frequency $1.473 \mathrm{GHz}$. (b) Gain absorption-efficiency pairs $\left(G, \eta_{\text {abs }}\right)$ of its lossy and lossless implementations for excitation frequencies ranging from $1.46 \mathrm{GHz}$ to $1.49 \mathrm{GHz}$. Solid arrows indicate the directions in which frequency increases. Inset: Zoom in to the maxima of their gain values. For comparison, the antenna gain which follows the upper bound (10) and the antenna gain for an ideal Huygens particle for $\eta_{\text {abs }}$ ranging from 0 to 1 are also provided.

of the lossless implementation approaches very closely its maximal value given by the forward scattering directivity: $D_{\text {scat }}\left(\widehat{\mathbf{k}}_{i}\right)=3.211$. Thus, it also can be considered optimal in the sense of maximizing the absorption.

On the other hand, it may be desirable, for instance, for various sensor applications to have significantly less visible antennas with higher absorption efficiency. Receiving antennas of this type are perfectly possible. From a Norton or Thevenin equivalent circuit perspective, it could be expected that increasing the port impedance should directly lead to a higher absorption efficiency. In fact, this strategy works perfectly well for simple wire antennas [19] and core-shell resonators [12]. However, equivalent circuit representations are only valid as long as the current distribution in the antenna is maintained. Unfortunately, for the coin antenna depicted in Fig. 4, changes in its port impedance modify the reflection coefficient at that

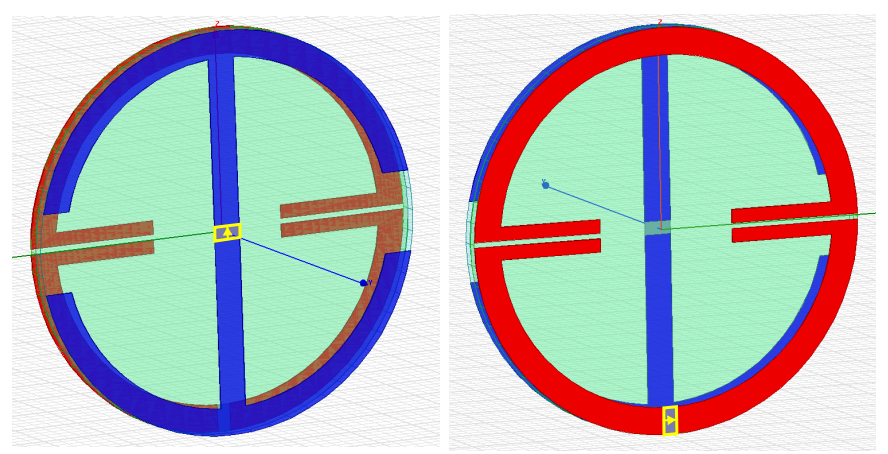

Fig. 6. Two-port coin antenna HFSS model. Front and back views. The schematic positions of the lumped ports are shown in yellow.

end of the CPS feed line. These modifications subsequently alter the field distributions that excite both the electric and magnetic resonators. This effect breaks the balance between the electric and magnetic resonators and, hence, ruins the forward-scattering characteristics. Therefore, the geometry of the coin antenna must be re-optimized for its operation at each desired, different absorption efficiency level.

\section{Coin Receiving Antenna: Two Ports}

To overcome this difficulty and to more clearly illustrate the performance of an antenna at different absorption efficiency levels, the feeding mechanism of the original coin antenna is modified so that its current distribution is less sensitive to changes in the port impedance. As depicted in Fig. 6, the antenna is now terminated at two lumped ports (marked in yellow): one placed at the center of the top-hat-loaded dipole resonator, and the other one placed in the middle of one of the capacitively loaded loops. The length of the ports is $1 \mathrm{~mm}$. The geometries of the resonators are exactly the same as in Fig. 2. In this two-port configuration, the coupling between both ports is negligible, i.e., $S_{21}<30 \mathrm{~dB}$ for all the following simulations. The lumped ports of the dipole and the open loop are characterized by the intrinsic impedances $C \times 1.5 \Omega$ and $C \times 5 \Omega$, respectively, where $C$ is used as a scaling factor to study the response of the receiving antenna to changes in its input impedance. In particular, $C=1$ corresponds to the matched configuration at which the gain is maximized.

Fig. 7(a) gives the H-plane scattering directivity pattern for the two-port coin antenna under its matched condition, $C=1$, at its resonant frequency, $1.504 \mathrm{GHz}$. Once again, the antenna concentrates all of its scattering in the forward direction with maximal directivity in excess of 3 . On the other hand, the FTBR in this case is only approximately 40 $(16 \mathrm{~dB})$. In order to analyze the response of the antenna to an induced port mismatch, Fig. 7(b) represents the antenna gain and its scattering directivity at the resonant frequency in the forward direction for impedance scaling factors $C$ ranging from 1 to 5 . As expected, the antenna gain monotonically decreases along with the $C$ factor due to the mismatch losses. However, it is also observed that the scattering directivity in the forward direction is mostly unaffected by the changes in the port impedance. This behavior confirms the robustness of the current distribution, including both electric and magnetic 


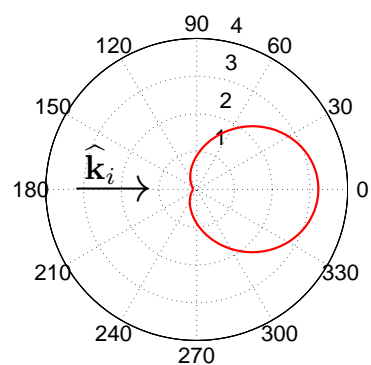

(a)

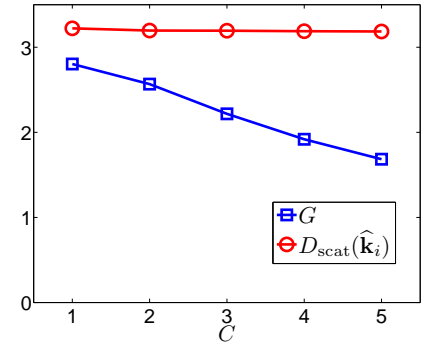

(b)
Fig. 7. HFSS simulated performance of the two-port coin antenna: (a) Hplane scattering directivity pattern under the matched condition, $C=1$, at its resonant frequency $1.504 \mathrm{GHz}$, (b) antenna gain and scattering directivity in the forward direction for impedance scaling factors $C$ ranging from 1 to 5 .

resonators, against impedance changes, for this two-port configuration. This property enables the operation of the two-port coin antenna at different absorption efficiency levels.

To illustrate this fact further, Fig. 8 represents the gain - absorption efficiency pair $\left(G, \eta_{\text {abs }}\right)$ as a function of the frequency for the two-port coin antenna port impedance when the $C$ impedance parameter ranges from 1 to 5 . As anticipated, increasing the $C$ impedance scaling factor increases the absorption efficiency of the antenna. Moreover, the antenna performance, as compared to the theoretical bound, is not degraded by the induced mismatch. Rather, the antenna performance approaches the upper bound as the port impedance increases. This fact can be more clearly appreciated in the inset of Fig. 8, which depicts a comparison of the upper bound (10) with the gain absorption-efficiency pairs $\left(G, \eta_{\mathrm{abs}}\right)$, at the maximal gain value for each $C$ value. We ascribe this behavior to the fact that, as the port impedance increases, the proportion of the power dissipated in the load to that lost in the copper and dielectric is enhanced, reducing the negative impact of the additional unwanted losses.

\section{CONCLUSIONS}

The optical theorem was used to derive upper bounds on the far-field cross-sections associated with general scatterers. These bounds were applied to understand the scattering and absorption properties of receiving antennas. It was shown via the optical theorem that energy conservation imposes the constraint that any extraction of power from an incident field via a scatterer, e.g., a receiving antenna, must be associated with the re-radiation of fields in the forward direction. The existence of this forwardly-directed scattered field imposes a correlation between the scattering and absorption phenomena and, hence, limits the amount of achievable absorption. Furthermore, the associated scattering resistance damps the excitation of currents in the scatterer and, thus, limits the amount of achievable scattering even in the ideal limit of no material dissipation [12], [13]. Previously derived bounds on the time-harmonic absorption and scattering cross-sections were reaffirmed independently by understanding these physical effects and their limitations. Moreover, this approach revealed novel upper bounds on the bistatic cross-section, i.e., the amount of scattering into a specific direction. These bounds led

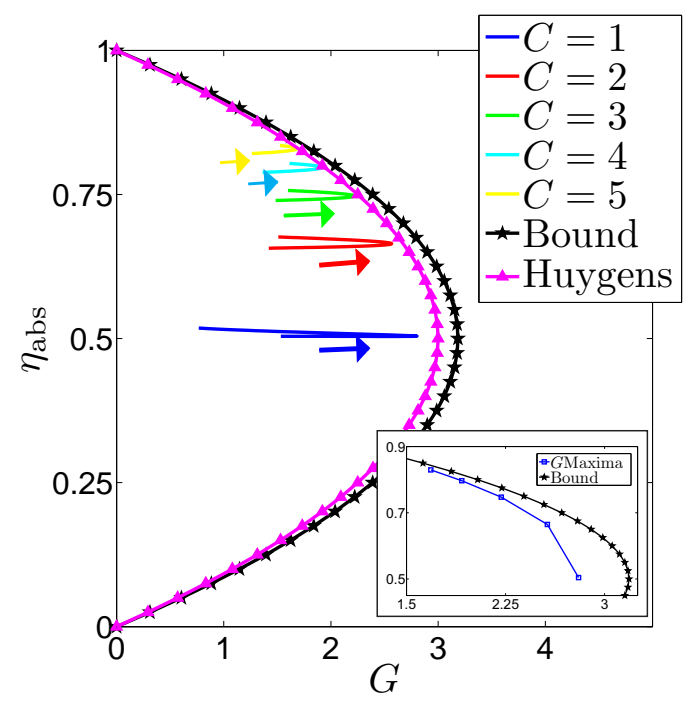

Fig. 8. HFSS simulated performance of the two-port coin antenna: Gain absorption-efficiency pairs $\left(G, \eta_{\text {abs }}\right)$ for excitation frequencies ranging from $1.49 \mathrm{GHz}$ to $1.53 \mathrm{GHz}$, and for impedance scaling factors $C$ ranging from 1 to 5 . Solid arrows indicate the directions in which the frequency is increasing. For comparison purposes, the upper bound (10) and the $\left(G, \eta_{\mathrm{abs}}\right)$ pairs for $\eta_{\text {abs }}$ ranging from 0 to 1 are also provided. Inset: Comparison of the gain absorption-efficiency pairs $\left(G, \eta_{\text {abs }}\right)$ at the maximal gain value for each $C$ value with the upper bound (10).

to the fact that the maximal bistatic cross-section can only be achieved in the forward scattering direction. Moreover, it was also found that the upper bound of the backscattering crosssection, of crucial importance for many practical applications, is four times smaller than the forward scattering maximum value. Furthermore, it was concluded that the backscattering upper bound can be reached with the excitation of only electric or magnetic multipoles, which was recognized as being a great advantage at optical frequencies where the magnetic responses of matter are weak.

The reported upper bounds were used to reveal the fact that receiving antennas must concentrate their scattered fields into the forward direction to maximize their absorption and scattering cross-sections, as well as their bistatic cross-section. It was demonstrated that this property is essential to achieve the maximum performance of a receiving antenna, such as a minimum scattering sensor. Understanding this constraint, we designed several metamaterial-inspired receiving antennas that exhibit Huygens source forward-scattering properties and showed that their ideal realizations actually reach the derived upper bounds. Issues that allow their realistic counterparts to only approach these bounds were highlighted. These Huygens source-based receiving antenna implementations further revealed that relatively simple designs, based only on the balanced combination of fundamental electric and magnetic dipole resonators rather than on higher order modes, offer performances that can very closely approach the physical bounds while being realizable.

\section{REFERENCES}

[1] C. G. Montgomery, R. H. Dicke, and E. M. Purcell, Principles of Microwave Circuits in Radiation Laboratory Series. New York: McGraw-Hill, 1948. 
[2] W. K. Kahn and H. Kurss, "Minimum-scattering antennas," IEEE Trans. Antennas Propag., vol. 13, no. 5, pp. 671-675, 1965.

[3] R. Green, "Scattering from conjugate-matched antennas," IEEE Trans. Antennas Propag., vol. 14, no. 1, pp. 17-21, 1966.

[4] A. Alù and N. Engheta, "Cloaking a sensor," Phys. Rev. Lett., vol. 102, no. 23, 233901, Jun. 2009.

[5] J. Vehmas, P. Alitalo, and S. A. Tretyakov, "Experimental demonstration of antenna blockage reduction with a transmission-line cloak," IET Microwaves Antennas Propag., vol. 6, no. 7, pp. 830-834, 2012.

[6] P. Alitalo, A. E. Culhaoglu, A. V. Osipov, S. Thurner, E. Kemptner, and S. A. Tretyakov, "Experimental characterization of a broadband transmission-line cloak in free space," IEEE Trans. Antennas Propag., vol. 60, no. 10, pp. 4963-4968, 2012.

[7] I. Liberal, I. Ederra, R. Gonzalo, and R. W. Ziolkowski, "A multipolar analysis of near-field absorption and scattering processes," IEEE Trans. Antennas Propag., vol. 61, no. 10, pp. 5184-5199, Oct. 2013.

[8] K. V. S. Rao, P. Nikitin, and S. Lam, "Antenna design for UHF RFID tags: A review and a practical application," IEEE Trans. Antennas Propag., vol. 53, no. 12, pp. 3870-3876, Dec. 2005.

[9] I. Liberal, I. Ederra, and R. Gonzalo, "Fe-Rich ferromagnetic wires for self-sensing materials," IEEE Trans. Microw. Theory Techn., vol. 60, no. 9, pp. 2752-2759, Sept. 2012.

[10] R. G. Newton, "Optical theorem and beyond," Am. J. Phys., vol. 44, no. 7, pp. 639-642, 1976.

[11] J. B. Andersen and A. Frandsen, "Absorption efficiency of receiving antennas," IEEE Trans. Antennas Propag., vol. 53, no. 9, pp. 28432849, Sept. 2005.

[12] I. Liberal and R. W. Ziolkowski, "Analytical and equivalent circuit models to elucidate power balance in scattering problems," IEEE Trans. Antennas Propag., vol. 61, no. 5, pp. 2714-2726, May 2013.

[13] I. Liberal, Y. Radi, R. Gonzalo, I. Ederra, S. A. Tretyakov, and R. W. Ziolkowski, "Least upper bounds of the power extracted and scattered by bi-anisotropic particles," In press, IEEE Trans. Antennas Propag., 2014.

[14] D. H. Kwon and D. Pozar, "Optimal characteristics of an arbitrary receive antenna," IEEE Trans. Antennas Propag., vol. 57, no. 12, pp. 3720-3727, Dec. 2009.

[15] M. Gustafsson, J. B. Andersen, G. Kristensson, and G. F. Pedersen, "Forward scattering of loaded and unloaded antennas," IEEE Trans. Antennas Propag., vol. 60, no. 12, pp. 5663-5668, 2012.

[16] R. W. Ziolkowski, P. Jin, and C.-C. Lin, "Metamaterial-inspired engineering of antennas," Proc. IEEE, vol. 99, no. 10, pp. 1720-1731, Oct. 2011.

[17] C. F. Bohren and D. R. Huffman, Absorption and Scattering of Light by Small Particles. New York: John Wiley \& Sons, 2008.

[18] C. A. Balanis, Antenna Theory: Analysis and Design, 3rd ed. New York: John Wiley \& Sons, 2012.

[19] A. Alù and S. I. Maslovski, "Power relations and a consistent analytical model for receiving wire antennas," IEEE Trans. Antennas Propag., vol. 58, no. 5, pp. 1436-1448, May 2010.

[20] Z. Ruan and S. Fan, "Superscattering of light from subwavelength nanostructures," Phys. Rev. Lett., vol. 105, no. 1, p. 013901, 2010.

[21] C. Sohl, M. Gustafsson, and G. Kristensson, "Physical limitations on broadband scattering by heterogeneous obstacles," J. Phys. A, vol. 40, no. 36, p. 11165, 2007.

[22] M. Gustafsson, C. Sohl, and G. Kristensson, "Physical limitations on antennas of arbitrary shape," Proc. Royal Soc. A., vol. 463, no. 2086, pp. 2589-2607, 2007.

[23] C. Sohl, M. Gustafsson, and G. Kristensson, "Physical limitations on metamaterials: restrictions on scattering and absorption over a frequency interval," J. Phys. D, vol. 40, no. 22, pp. 7146-7151, 2007.

[24] C. Sohl, C. Larsson, M. Gustafsson, and G. Kristensson, "A scattering and absorption identity for metamaterials: Experimental results and comparison with theory," J. Appl. Phys., vol. 103, no. 5, pp. 4906-4910, 2008.

[25] M. Gustafsson, "Time-domain approach to the forward scattering sum rule," Proc. Royal Soc. A, vol. 466, no. 2124, pp. 3579-3592, 2010.

[26] F. Monticone and A. Alù, "Do cloaked objects really scatter less?" Phys. Rev. $X$, vol. 3, p. 041005, Oct 2013

[27] R. F. Harrington, "On the gain and beamwidth of directional antennas," IRE Trans. Antennas Propag., vol. 6, no. 3, pp. 219-225, Jul. 1958.

[28] — , Time-Harmonic Electromagnetic Fields. New York: McGrawHill, 1961.

[29] L. D. Landau and E. M. Lifshitz, Electrodynamics of Continuous Media. New York: Pergamon Press, 1960.
[30] D. H. Kwon, "On the Radiation $Q$ and the gain of crossed electric and magnetic dipole moments," IEEE Trans. Antennas Propag., vol. 53, no. 5, pp. 1681-1687, 2005.

[31] A. D. Yaghjian, T. H. O'Donnell, E. E. Altshuler, and S. R. Best, "Electrically small supergain end-fire arrays," Radio Sci., vol. 43, no. 3, p. RS3002, 2008.

[32] A. D. Yaghjian, "Increasing the supergain of electrically small antennas using metamaterials," in EuCAP 2009. 3rd European Conf. Antennas Propag., Berlin, Germany, 2009, pp. 858-860.

[33] R. P. Haviland, "Supergain antennas: possibilities and problems," IEEE Antennas Propag. Mag., vol. 37, no. 4, pp. 13-26, 1995.

[34] J. L. Volakis, C.-C. Chen, and K. Fujimoto, Small Antennas: Miniaturization Techniques \& Applications. New York: McGraw-Hill, 2010.

[35] M. Kerker, D. S. Wang, and C. L. Giles, "Electromagnetic scattering by magnetic spheres," J. Opt. Soc. Am., vol. 73, no. 6, pp. 765-767, Jun 1983.

[36] I. Liberal, I. Ederra, R. Gonzalo, and R. W. Ziolkowski, "Induction theorem analysis of resonant nanoparticles: Design of a huygens source nanoparticle laser," Phys. Rev. Applied, vol. 1, p. 044002, May 2014.

[37] P. Jin and R. W. Ziolkowski, "Metamaterial-inspired, electrically small Huygens sources," IEEE Antennas Wireless Propag. Lett., vol. 9, pp. 501-505, 2010.

[38] T. Niemi, P. Alitalo, A. Karilainen, and S. A. Tretyakov, "Electrically small Huygens source antenna for linear polarisation," IET Microwaves Antennas Propag., vol. 6, no. 7, pp. 735-739, 2012.

[39] P. Alitalo, A. O. Karilainen, T. Niemi, C. R. Simovski, and S. A. Tretyakov, "Design and realisation of an electrically small Huygens source for circular polarisation," IET Microwaves Antennas Propag., vol. 5 , no. 7 , p. 783, 2011.

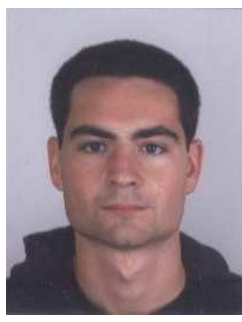

Iñigo Liberal received the Engineer's (2009), M.Sc. (2010) and Ph. D. (2013), magna cum laude, degrees in Telecommunication Engineering from the Public University of Navarra (UPNA), Spain. He was a visiting student at Delft University of Technology (Delft, The Netherlands), and a visiting researcher at Aalto University (Helsinki, Finland) and the University of Arizona (Tucson, USA).

He currently is a postdoctoral fellow at the Antenna Group, Department of Electrical and Electronic Engineering, Public University of Navarra (UPNA), Spain. His main research interests are in the fields of scattering and antenna theory, with emphasis on electromagnetic manipulation and trapping, electrically small antennas and artificial electromagnetic materials.

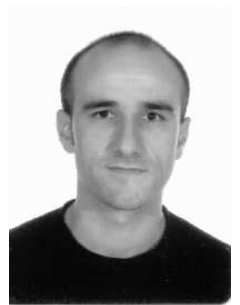

Iñigo Ederra was born in Isaba, Navarra, Spain in 1972. He received the Ingeniero de Telecomunicación and Ph.D. degrees from the Universidad Pública de Navarra, Pamplona, Spain, in 1996 and 2004, respectively.

In 1997, he joined the Microwave and Millimetre Wave Group, Universidad Pública de Navarra. From 1999 to 2000 he was with the European Space Research and Technology Centre (ESTEC), ESA, Noordwijk, The Netherlands, where he was working on Electromagnetic Bandgap materials and their applications in the field of antennas. Since 2001 he is with the Antenna Group, Universidad Pública de Navarra. From June to October 2002 he was visitor scientist at the Rutherford Appleton Laboratory, Chilton, Didcot, UK, participating in the Startiger project.

He was co-recipient of the LAPC 2006 and IWAT 2007 best paper award. His research interests are in the field of Electromagnetic Bandgap materials and Metamaterials and their applications in microwave and millimetre wave components and antennas. 
This is the author's version of an article that has been published in this journal. Changes were made to this version by the publisher prior to publication.

The final version of record is available at http://dx.doi.org/10.1109/TAP.2014.2359206

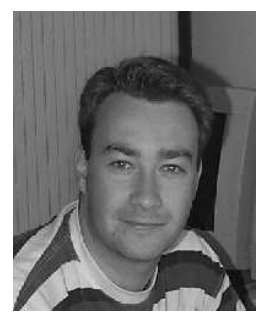

Ramón Gonzalo (S'95) was born on July 15, 1972 in Logroño, La Rioja. He received the M.Sc. and the $\mathrm{Ph}$. D. degree in Ingeniero de Telecomunicación both with honors, from the Public University of Navarra (UPNa), Spain.

Since October 1995 he is with the Antenna Group at the Electrical and Electronic Engineering Department in UPNa where he currently is Associate Professor. From September 1997 to December 1998, he was joined as Research Fellow to the Antenna Section in ESA-ESTEC where he was involved in the modelling and design of electromagnetic crystal devices at microwave and millimetre wave frequencies. He has been involved in more than 25 research project, European, and National level acting as co-ordinator in several of them. In particular he has been co-ordinator of 5 projects funded by the European Space Agency, 2 projects in the framework of the European Commission and several of them funded by the Spanish Minister of Science. He has more than 50 journal publications in peer review magazines and 100 conference papers related to his research lines. From January 2006 untill April 2008 he was acting as sub-director of the Engineering Faculty and from April 2008 he is acting as Head of Electrical and Electronic Engineering Department.

His current area of research is in the field of Terahertz technologies, subsystems and devices, Electromagnetic Band Gap technology with emphasis on space antenna applications, design of waveguide transmission lines and corrugated horn antennas.

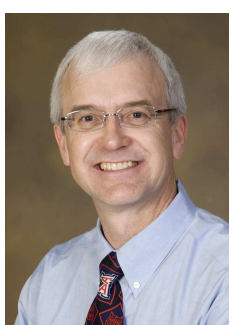

Richard W. Ziolkowski (M'87-SM'91-F'94) received his Sc.B. degree in physics, magna cum laude with honors, from Brown University (1974), and his M.S. (1975) and Ph.D. (1980) degrees in physics from the University of Illinois at UrbanaChampaign. $\mathrm{He}$ is the Litton Industries John M. Leonis Distinguished Professor in the Department of Electrical and Computer Engineering at the University of Arizona. He is also a Professor in the College of Optical Sciences at the University of Arizona. He is a Distinguished Adjunct Professor to King Abdulaziz University (KAU), Jeddah, Saudi Arabia. He was awarded an Honorary Doctorate, Doctor Technish Honoris Causa, from the Technical University of Denmark (DTU) in 2012. He was the Computational Electronics and Electromagnetics Thrust Area Leader in the Engineering Research Division at the Lawrence Livermore National Laboratory before joining the University of Arizona in 1990 .

Prof. Ziolkowski is a Fellow of both the Institute of Electrical and Electronics Engineers (IEEE) and the Optical Society of America (OSA). He served as the President of the IEEE Antennas and Propagation Society in 2005. He is also actively involved with the URSI, OSA and SPIE professional societies. He and Prof. Nader Engheta, University of Pennsylvania, are CoEditors of the best-selling 2006 IEEE-Wiley book, Metamaterials: Physics and Engineering Explorations. 